\title{
Burden and outcome of community-acquired pneumonia in adult patients admitted to National Referral Hospital, Bhutan
}

\author{
Sonam Choki ${ }^{1}$, Chhabi Lal Adhikari², Dhrupthob Sonam³, Sonam Chhoden $\mathrm{R}^{4}$ \\ ${ }^{1,4}$ Faculty of Postgraduate Medicine, Khesar Gyalpo University of Medical Sciences of Bhutan, Thimphu, Bhutan \\ ${ }^{2,3}$ Department of General Practice, Jigme Dorji Wangchuck National Referral Hospital, Thimphu, Bhutan
}

\begin{abstract}
Introduction: Community-acquired pneumonia (CAP) is one of the leading causes of morbidity and mortality globally with the highest burden being reported from Asia. In Bhutan, CAP was reported to be one of the top five causes of mortality and one of the top ten causes of morbidity. Methods: A prospective observational study was conducted in adult patients diagnosed with CAP who were admitted to the medical wards in the National Referral Hospital, from February 2020 - February 2021. Purposive sampling technique was used to recruit the patients. The burden and outcome of the CAP was evaluated and the predictive capability of CURB-65 (Confusion, urea, respiratory rate, blood pressure and age $\geq 65$ ) score to predict mortality as an outcome in these patients was assessed. Results: The inpatient burden of CAP was found to be $4.7 \%$. The mortality was observed as $7.8 \%$. There were 15.7 $\%$ of patients who were managed in the intensive care unit out of which $5.9 \%$ patients needed mechanical ventilation. The mean hospital length of stay of these patients was 13 days. The sensitivity, specificity, Positive Predictive Value (PPV) and Negative Predictive Value (NPV) of CURB-65 score to predict death as an outcome in these patients were $87.5 \%, 43.6 \%, 11.7 \%$ and $97.6 \%$ respectively. Conclusions: The inpatient burden and outcome of CAP in the National Referral Hospital is of concern with mortality as high as $7.8 \%$. The CURB-65 score can be used as a supplement to the clinical judgement to assess the severity of the disease and make appropriate management decisions.
\end{abstract}

Keywords: Burden; Community acquired pneumonia; CURB-65; Mortality.

\section{INTRODUCTION}

Community-acquired pneumonia is an infectious disease that is one of the leading causes of morbidity and mortality. The 2019 Global Burden of Diseases study demonstrated that there were 489 million incident cases and 11 million prevalent cases of lower respiratory tract infections including pneumonia. It had contributed to 2.49 million deaths worldwide and 97.2 million disability adjusted life years ${ }^{1}$. CAP has considerable impact on the health care system. A study done in Asian countries such as Malaysia, Indonesia and the Philippines found that the cost of admitting patients with CAP was between 254 to 1208 US dollars ${ }^{2}$. A study reported that the overall mortality in patients with CAP in eight Asians countries was $7.3 \%{ }^{3}$. According to the Annual Health Bulletin of Bhutan, 2020, CAP was reported to be one of the top five causes of mortality and one of the top ten causes of morbidity ${ }^{4}$. Assessing the severity of the illness and making the appropriate management decisions can minimize unnecessary hospital admission and exhaustion of resources,

Corresponding author:

Sonam Choki

drchoki.sonam@gmail.com but also prioritize patients requiring prompt intensive unit care and thereby reduce the likelihood of complications and death. There are several severity assessment tools and one such tool is the CURB-65 score introduced by Lim, et $\mathrm{al}^{5}$. The CURB-65 stands for Confusion, Urea $>7 \mathrm{mmol} / \mathrm{L}$, Respiratory Rate $>30$ breaths, Blood pressure (systolic blood pressure $<90 \mathrm{mmHg}$ or diastolic blood pressure $<60 \mathrm{mmHg}$ ) and Age $\geq 65$ years $^{5}$ and the score is calculated by giving 1 point each to the aforementioned prognostic factors. Using the CURB-65 score, patients can be classified into low, intermediate and high-risk groups ${ }^{5,6}$.

Currently in Bhutan, there are no studies on CAP in adults nor the use of the severity assessment tools. We do not know the burden nor the outcome of this disease, and there is no consensus on the use of severity tools among clinicians. The CURB-65 score is one such tool that can be used in a resource poor country like Bhutan. We studied the burden of CAP in adult patients admitted in the National Referral Hospital and described the outcome in terms of discharge, intensive care unit admissions and mechanical ventilation, length of hospital stay and death. We studied the ability of the CURB-65 score to predict death as an outcome in these patients. 


\section{METHODS}

After acquiring approval from the Research Ethics Board of Health (Ref. No. REBH/Approval/2019/061) dated $12^{\text {th }}$ December 2019, a prospective observational study conducted in the medical wards of the National Referral Hospital, Thimphu, Bhutan. Inclusion criteria was adults aged $\geq 18$ years, cough for 21 days or less with at least one of the symptoms such as fever, sputum production, breathlessness, chest pain or discomfort, and the diagnosis confirmed by chest radiography. Patients who developed pneumonia 48 hours after hospital admission, those patients who had a history of hospital admission in the preceding 14 days and patients diagnosed with pulmonary tuberculosis were excluded from the study.

Consecutive sampling of all patients admitted to the medical Wards of the National Referral Hospital, Thimphu, Bhutan from $15^{\text {th }}$ February 2020 to $15^{\text {th }}$ February 2021, fulfilling the inclusion criteria were enrolled in the study.

A structured questionnaire was designed which was pretested among 20 patients, reliability of it was tested using the Cronbach's alpha and validated it. The questionnaire was used to collect the information of the patients, to calculate the CURB-65 score and follow up on the outcome of the patients.

Patients and their care-givers were interviewed to obtain information on age, gender, symptoms and comorbid conditions. At the time of admission, the patient's mental status was assessed by the admitting physician or the investigator and confusion was defined as disorientation in time or place or person. The first recorded parameters such as the blood pressure which was measured using an automatic blood pressure monitor device and respiratory rate measured at the time of admission were used. The measurements were recorded in the questionnaire. Blood urea levels were extracted from the investigation records of the patient which were sent at the time of admission. Results of the patients' sputum investigations were obtained from the medical records of the patient.

With the first recorded parameters of the patient such as mental status, blood urea levels, respiratory rate, blood pressure and the age of the patient recorded in years, the CURB-65 score of each individual patient was calculated. The CURB-65 score was calculated by giving 1 point each to the afore mentioned prognostic factors.

Using the CURB-65 score, the patients were stratified as low, intermediate and high-risk groups for mortality prediction. Patients with CURB-65 score of 0-1 were classified as low risk, those with CURB-65 score of 2 as intermediate risk and those with CURB-65 score of 3-5 as high-risk group ${ }^{5,6}$.

The patients were then followed up from the time of admission till 30 days to ascertain the outcome of CAP and time period of resolution of the presenting symptoms of patients. Patients were followed up on day $7^{\text {th }}, 15^{\text {th }}$ and $30^{\text {th }}$ day and resolution of the presenting symptoms were noted on the above-mentioned time period respectively. If the patients were discharged during the follow up period, the patient or their care giver were interviewed over the telephone and inquired about the resolution of patient's presenting symptoms and adverse outcomes such as death in the community were ascertained.

Data was entered in electronic form using EpiData V3.1 and double entry was done to check for accuracy. Data was analyzed using EpiData Analysis V2.2.2.183.

A 2x2 table was created and MedCalc app was used to calculate the sensitivity, specificity, positive predictive value and negative predictive value of CURB-65 score to predict death as an outcome.

Sensitivity is defined as the proportion of positives (deaths) which were correctly predicted by the CURB-65 score $\geq 2$ or the probability of a positive test, also known as the true positive rate (=true positives / [true positive + false negatives] $)^{7}$. Specificity is defined as the proportion of negatives (survived) which were correctly predicted by the CURB- 65 score $<2$ or the probability of a negative test, also known as the true negative rate $(=\text { true negatives / [true negative }+ \text { false positives] })^{7}$.

PPV is the probability that a patient has the disease (deaths) given that the test results are positive. It is given by the ratio of true positives / (true positives + false positives $)^{8}$.

NPV is the probability that a patient does not have the disease (survived) given that the test results are indeed negative. It is given by the ratio of true negative / (true negative + false negatives $)^{8}$.

\section{RESULTS}

There were a total of 2155 patients admitted in the medical wards of the national referral hospital during the study period. A total of 102 patients who fulfilled the inclusion criteria were enrolled in the study. The in patient burden of CAP in the medical wards of the national referral hospital was $4.7 \%$ during the study period. The mean age of patients was $57.3 \pm 18.2$ among the 102 patients with the age ranging from 19-96 years. Thirty nine percent of the patients were within the age group of 44-65 years and male patients were comparatively more than females(52\% vs 48\%). Thirty patients $(29.4 \%)$ had no known comorbid conditions whereas $72(70.6 \%)$ patients had one or more than one comorbid condition. The comorbidities observed among the patients were cardiovascular diseases $(44.8 \%)$, pre-existing lung disease $(20 \%)$ followed by diabetes and chronic kidney disease (12.4\% each). The characteristics of the patients are shown in Table 1.

\section{Presenting symptoms and resolution of symptoms}

The commonest presenting symptom was cough (100\%), followed by breathlessness $(96.8 \%)$ and expectoration $(66.67 \%)$ as shown in Table 1. During the follow up period, $39.3 \%$ of patients reported resolution of their presenting symptoms by day $7,39.2 \%$ by day $15,12.7 \%$ by 30 days and the rest with persistence of symptoms beyond 30 days as shown in Figure 1. 
Table 1. Characteristics of patients with CAP* admitted in the Medical Wards of the National Referral Hospital, Bhutan from $2020-2021(n=102)$

\begin{tabular}{|c|c|c|}
\hline Characteristics & $\mathrm{n}$ & $(\%)$ \\
\hline \multicolumn{3}{|l|}{ Age Group } \\
\hline $19-23$ & 2 & 2 \\
\hline $24-43$ & 24 & 23.5 \\
\hline $44-65$ & 39 & 38.2 \\
\hline$>65$ & 37 & 36.3 \\
\hline \multicolumn{3}{|l|}{ Sex } \\
\hline Male & 53 & 52 \\
\hline Female & 49 & 48 \\
\hline \multicolumn{3}{|l|}{ Presenting Symptoms } \\
\hline Cough & 102 & 100 \\
\hline Fever & 62 & 60.8 \\
\hline Breathlessness & 98 & 96.1 \\
\hline Expectoration & 68 & 66.6 \\
\hline Chest pain or discomfort & 41 & 40.2 \\
\hline \multicolumn{3}{|l|}{ Co-morbidities } \\
\hline Pre-existing lung diseases & 21 & 20 \\
\hline Diabetes mellitus & 13 & 12.4 \\
\hline Autoimmune Disease & 1 & 0.96 \\
\hline $\mathrm{CLD}^{\dagger}$ & 5 & 4.7 \\
\hline CKD & 13 & 12.4 \\
\hline $\mathrm{CVD}^{\S}$ & 1 & 44.8 \\
\hline Others & 5 & 4.7 \\
\hline Number of Comorbidity & & 29.4 \\
\hline None & 30 & 70.6 \\
\hline$\geq 1$ comorbidity & 72 & \\
\hline
\end{tabular}

*Community-acquired pneuomonia,

†Chronic liver disease, $\neq$ Chronic kidney disease, \$Cardiovascular diseases

There were 58 sputum samples sent for gram stain and culture, out of which positive yield was reported in 12 samples, the most common organism isolated was Klebsiella pneumoniae as shown in Figure 2.

\section{Risk scoring of patients using CURB-65 Score}

Using the CURB-65 score at the time of hospital admission, 41.2 $\%$ of patients were stratified as low risk. Those patients with CURB-65 score of 1 and 2 had at least one or more than one comorbidity than those with higher CURB-65 scores of 3 or 4 . There were no deaths noted in patients who had a CURB- 65 score of 0 . Four patients died with CURB- 65 score of 2 . The CURB-65

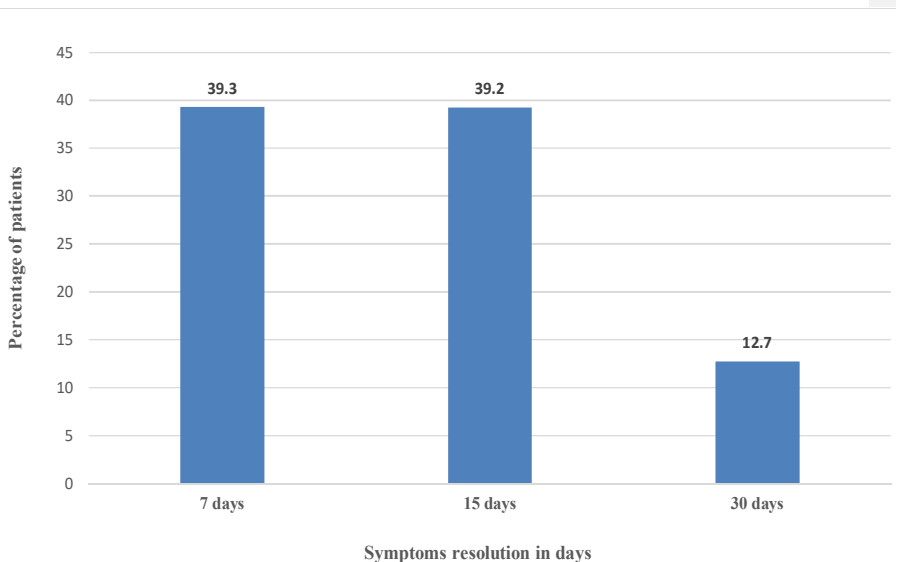

Figure 1. Resolution of presenting symptoms in patients with CAP at $7^{\text {th }}, 15^{\text {th }}$ and $30^{\text {th }}$ day follow up period, admitted in medical wards in National Referral Hospital, Bhutan from 2020 - $2021(n=102)$

Organism Isolate from sputum culture

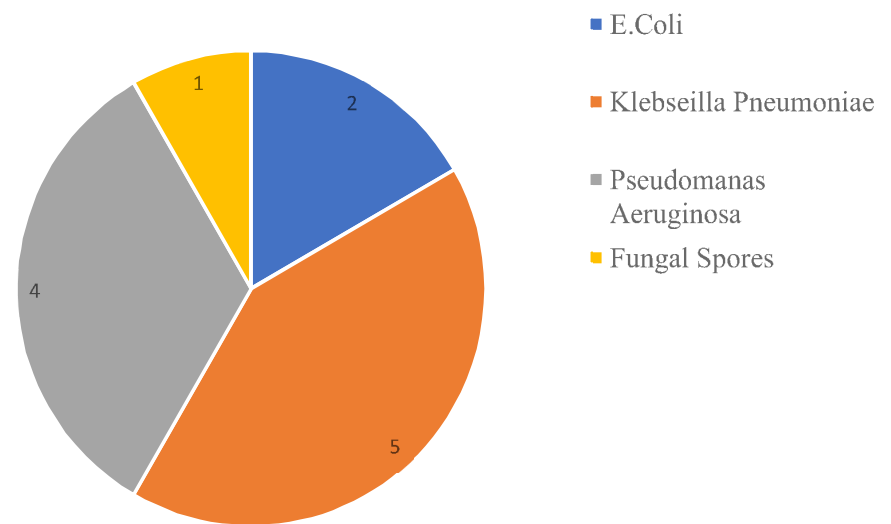

Figure 2. Etiological microorganisms identified from 12 positive sputum cultures of patients with CAP admitted to the National Referral Hospital, Thimphu, Bhutan from 2020 $-2021$

score with risk stratification, number of comorbid conditions of patients and mortality in patients with CAP is shown in Table 2.

CURB-65 score with outcome variable shown in table 2 was used to calculate the sensitivity, specificity, positive predictive value and negative predictive value of the CURB-65 score to predict mortality as an outcome. The predictive capability of CURB-65 score to predict mortality as an outcome is shown in Table 3.

Sensitivity of CURB-65 score to predict death as an outcome was $87.5 \%$ (47.32-99.7CI) with 11.7\% PPV (8.8 - 15.3 CI) as shown in Table 4.

Outcomes of patients with community acquired pneumonia At the end of the follow up period, 94 patients were discharged from the hospital and 8 deaths were noted. Out of the 16 patients 
Table 2. Risk stratification, number of comorbid conditions and mortality in patients with CAP* admitted to the National Referral Hospital, Bhutan from 2020 - $2021(n=102)$

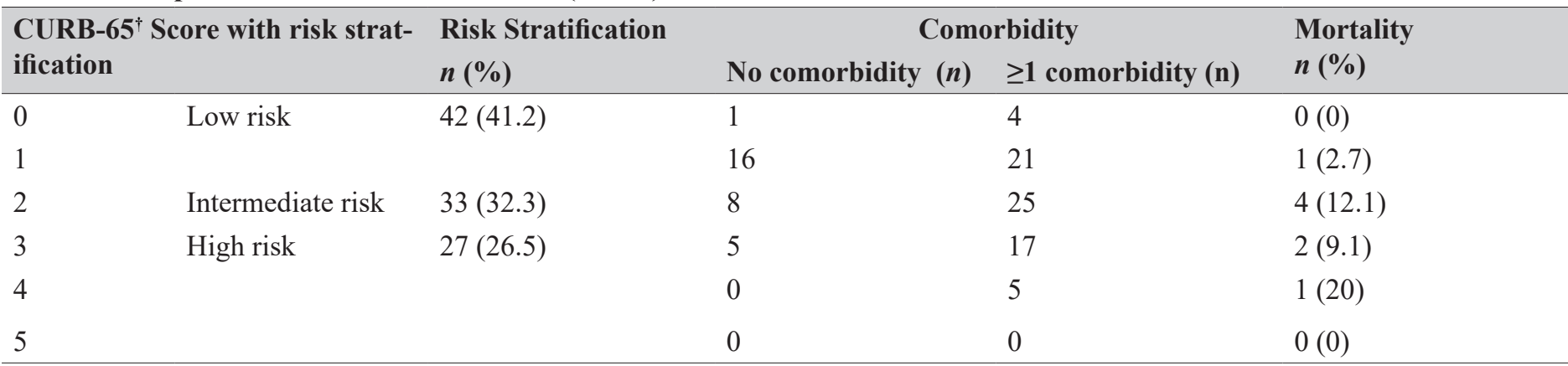

*Community-acquired pneumonia, ${ }^{\dagger}$ Confusion, Urea $>7 \mathrm{mmol} / \mathrm{L}$, Respiratory Rate $>30$ breaths, Blood pressure (systolic blood pressure $<90 \mathrm{mmHg}$ or diastolic blood pressure $<60 \mathrm{mmHg}$ ) and Age $\geq 65$ year

Table 3. CURB-65* score and outcome of patients with ${ }^{\dagger}$ CAP admitted to the Medical Wards of the National Referral Hospital, Bhutan from $2020-2021(n=102)$

\begin{tabular}{lll}
\hline CURB - 65* score & Number of Deaths & Number of Survived \\
$\geq 2$ & 7 & 53 \\
$<2$ & 1 & 41 \\
\hline
\end{tabular}

*Confusion, Urea $>7 \mathrm{mmol} / \mathrm{L}$, Respiratory Rate $>30$ breaths, Blood pressure (systolic blood pressure $<90 \mathrm{mmHg}$ or diastolic blood pressure $<60 \mathrm{mmHg}$ ) and Age $\geq 65$ year, Community-acquired pneumonia

Table 4. The estimates and corresponding $95 \% \mathrm{CI}^{*}$ for Sensitivity, Specificity, PPV ${ }^{\star}$ and NPV of CURB-65 ${ }^{\S}$ score to predict death as an outcome in patients with "CAP admitted to the Medical Wards of the National Referral Hospital, Bhutan from 2020 - 2021 $(n=102)$

\begin{tabular}{ll}
\hline Variables & $\%(\mathbf{C I})$ \\
\hline Sensitivity & $87.5(47.3-99.7)$ \\
Specificity & $43.6(33.4-54.2)$ \\
$\mathrm{PPV}^{\dagger}$ & $11.7(8.8-15.3)$ \\
$\mathrm{NPV}^{\ddagger}$ & $97.6(86.6-99.6)$ \\
\hline
\end{tabular}

${ }^{*}$ Confidence Interval, ${ }^{*}$ Positive predictive value, ${ }^{*}$ Negative predictive value, ${ }^{s}$ Confusion, Urea $>7 \mathrm{mmol} / \mathrm{L}$, Respiratory Rate $>30$ breaths, Blood pressure (systolic blood pressure $<90 \mathrm{mmHg}$ or diastolic blood pressure $<60 \mathrm{mmHg}$ ) and Age $\geq 65$ year, "Community-acquired pneumonia

who were managed in the ICU, 6 patients needed mechanical ventilation as shown in Figure 3.

Patients with comorbidities (70) managed in the intensive care unit (ICU) had a higher CURB-65 score on admission and longer lengths of hospital stay than the patients manged in the medical wards as shown in Table 5.

On comparison of the groups of patients who had survived and died, the mean age of patients who died was 59.7 versus 27.1 in those who survived and all of the patients who died had at least one or more than one comorbidity. The mean CURB-

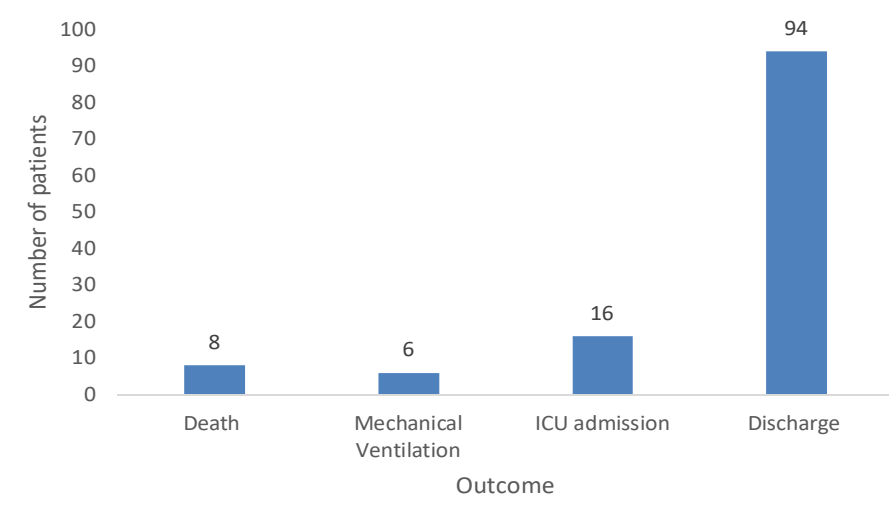

Figure 3. Outcome of patients with CAP admitted to the medical wards of National Referral Hospital, Bhutan from 2020 - $2021(n=102)$

Table 5. CAP* patients with comorbidities managed in the $\mathrm{ICU}^{\dagger}$ and the medical ward of the national referral hospital, Bhutan from $2020-2021(n=70)$

\begin{tabular}{|c|c|c|}
\hline Variables & ICU Admission & Medical ward \\
\hline \multicolumn{3}{|l|}{ Comorbidity } \\
\hline Lung Disease & 2 & 19 \\
\hline Diabetes & 2 & 11 \\
\hline $\mathrm{CVD}^{\sharp}$ & 2 & 33 \\
\hline $\mathrm{CKD}^{\S}$ & 0 & 13 \\
\hline $\mathrm{CLD}^{\|}$ & 1 & 4 \\
\hline Autoimmune & 0 & 1 \\
\hline Others & 0 & 2 \\
\hline $\begin{array}{l}\text { Mean age of patients ( } \pm \\
\left.\text { SD }^{\ddagger}\right)\end{array}$ & $56.3 \pm 18.2$ & $57.5 \pm 18.3$ \\
\hline $\begin{array}{l}\text { Mean CURB-65 score }( \pm \\
\left.\text { SD }^{\llbracket}\right)\end{array}$ & $2.68 \pm 0.7$ & $1.67 \pm 0.9$ \\
\hline $\begin{array}{l}\text { Mean length of stay }( \pm \\
\text { SD") }\end{array}$ & $24.2 \pm 15.6$ & $10.9 \pm 7.1$ \\
\hline \multicolumn{3}{|c|}{$\begin{array}{l}{ }^{*} \text { Community-acquired pneumonia, Intensive Care Unit, } \\
\text { "Cardiovascular diseases, SChronic kidney disease, "Chronic } \\
\text { liver disease. Others included blood and solid organ } \\
\text { malignancy, "Standard Deviation }\end{array}$} \\
\hline
\end{tabular}


Table 6. Comparison of variables in patients with CAP* who survived and died in the National Referral Hospital, Thimphu, Bhutan from 2020 - $2021(n=102)$

\begin{tabular}{|c|c|c|c|}
\hline Variable & $\begin{array}{l}\text { Survival } \\
n(\%)\end{array}$ & $\begin{array}{l}\text { Death } \\
n(\%)\end{array}$ & $p$ - value \\
\hline \multicolumn{4}{|l|}{ Gender } \\
\hline Male & $47(50)$ & $6(75)$ & 0.174 \\
\hline Female & $47(50)$ & $2(25)$ & \\
\hline $\begin{array}{l}\text { Mean age }\left( \pm \mathrm{SD}^{\dagger}\right) \text { in } \\
\text { years }\end{array}$ & $27.1 \pm 18.4$ & $59.7 \pm 16.2$ & \\
\hline $\begin{array}{l}\text { Mean CURB-65 score } \\
\left( \pm \mathrm{SD}^{\dagger}\right)\end{array}$ & $1.78 \pm 0.95$ & $2.37 \pm 0.91$ & \\
\hline \multicolumn{4}{|l|}{$\begin{array}{l}\text { Number of comorbid- } \\
\text { ities }\end{array}$} \\
\hline None & 30 (31.9) & $0(0.0)$ & 0.274 \\
\hline$\geq 1$ & $64(68.1)$ & $8(100)$ & \\
\hline \multicolumn{4}{|l|}{ Comorbid conditions } \\
\hline None & $30(31.9)$ & $0(0.0)$ & 0.000 \\
\hline Lung disease & $18(19.1)$ & $1(12.5)$ & \\
\hline $\mathrm{CVD}^{\S}$ & $19(20.2)$ & $3(37.5)$ & \\
\hline $\mathrm{CKD}^{\|}$ & $9(9.6)$ & $0(0.0)$ & \\
\hline CLD & $3(3.2)$ & $2(25)$ & \\
\hline Diabetes mellitus & $13(13.8)$ & $0(0.0)$ & \\
\hline Autoimmune disease & $1(1.1)$ & $0(0.0)$ & \\
\hline Others & $1(1.1)$ & $2(25)$ & \\
\hline
\end{tabular}

${ }^{*}$ Community-acquired pneumonia, ${ }^{\prime S t a n d a r d ~ D e v i a t i o n, ~}{ }^{*}$ Confusion, Urea $>7 \mathrm{mmol} / \mathrm{L}$, Respiratory Rate $>30$ breaths, Blood pressure (systolic blood pressure $<90 \mathrm{mmHg}$ or diastolic blood pressure < $60 \mathrm{mmHg}$ ) and Age $\geq 65$ year, ${ }^{\S}$ Cardiovascular diseases, "Chronic kidney disease, "Chronic liver disease. Others included blood and solid organ malignancy

65 score of those who died was 2.37 versus 1.78 in those who survived as shown in Table 6.

\section{DISCUSSION}

In this study, the mean age of patients was 57.3 which is similar to the findings reported by studies done in Asian countries ${ }^{3}$. CAP was more prevalent in the middle age and in the elderly which is consistent to the findings reported in studies in India, Spain and Europe ${ }^{9-11}$. The majority of participants were male patients in this study which is also consistent with global reports on sexbased differences in the prevalence and incidence of $\mathrm{CAP}^{3,9,12}$. The plausible explanation for these findings could be because of risk factors such as comorbid conditions, smoking, alcohol consumption and exposure to dust which are common in this age group and in males. Greater understanding of the types of individuals who are at risk of CAP can help ensure that the interventions to reduce the risk of infection and burden of the disease are targeted appropriately ${ }^{13}$.

The common symptoms reported by patients in this study were breathlessness, expectoration followed by fever. These findings were similar to those reported from Asia and European country such as Iceland ${ }^{3,14}$.

In this study, 68 patients reported to have expectoration at the time of admission during their interview but only 58 patients were able to produce sputum for evaluation. On evaluation of these sputum samples there was a poor yield of sputum culture with positive isolates reported in only 12 sputum samples out of 58 sputum samples that had been sent for investigation. The presenting symptoms and outcome of these patients in this study was similar irrespective of the microorganism identified as the etiology of CAP, these observations were consistent with observations made by Bjarnason, et $\mathrm{al}^{15}$. With these findings, if we follow the recommendations from the American Thoracic Society, the authors recommend obtaining of pretreatment gram stain and culture of respiratory secretions in patients who are identified as severe CAP, those being empirically treated for Methicillin Resistant Staphylococcus aureus (MRSA) or Pseudomonas, those patients who had been previously infected with MRSA or Pseudomonas and those who were hospitalized and received parenteral antibiotics in the last 90 days. It has been found that the use of sputum gram stain and culture alone or in combination with other microbiological testing did not demonstrate better patient outcomes and the overall poor yield of sputum evaluation for detecting organisms causing CAP, limits its impact on the management and patient outcomes ${ }^{14}$.

More than $70 \%$ of the patients in this study had at least one or more than one comorbidity which is consistent with observations made by Rivero, et al where increased rates of pneumonia are seen in these patients ${ }^{9}$. The common comorbidities among these patients were cardiovascular diseases and lung diseases which are consistent to findings reported by studies done in Asian countries such as India and Singapore, European countries such as Switzerland, UK, New Zealand and Netherland 11,16-18. These observations highlight the importance and need for appropriate measures for prevention and management of CAP in patients with these comorbid conditions to reduce morbidity and mortality associated with CAP.

The mortality in this study was $7.8 \%$ and $15.7 \%$ were managed in the ICU out of which $5.9 \%$ needed mechanical ventilation. The mortality in this study is lower compared to studies from Singapore and Iceland where they had large sample size and inclusion of more elderly patients in their study whereas the rates of ICU admissions and ventilation, and the mean length of hospital stay was lower in Singapore and Iceland. These differences could be because of the differences in the management approaches, systemic factors and threshold for ICU admission, criteria for transfer out of the ICU and discharge from the hospital ${ }^{15,16}$. The longer mean hospital length of stay in this study could be due to factors such as age of the patient, worsening of pre-existing comorbid conditions, ICU admission 
and mechanical ventilation, development of complications of pneumonia and, social factors of the patients and the caregivers. These factors were not taken into consideration or measured in this study.

When we compared the groups of patients who had survived and died, the mean age of patients who died was 59.7 versus 27.1 in those who survived and all of the patients who died had at least one or more than one comorbidity. The mean CURB65 score of those who died was 2.37 versus 1.78 in those who survived. Such findings highlight the risk of mortality in CAP with increasing age, those with comorbid conditions and higher CURB-65 score.

Using the CURB-65 score, upon risk stratification of patients at the time of hospital admission, we found that majority $(41.2 \%)$ of the patients were identified as low risk group (CURB65 score of $0-1)$. These patients could have been considered for home-based care management which might have reduced the inpatient burden of the disease ${ }^{6}$.

When we look at the mortality in each of the CURB65 scores, there were no deaths noted in patients who had a CURB-65 score of $0,12.5 \%$ of patients died who had a CURB65 score of $1,50 \%$ of patients with CURB-65 score of 2, 25\% with CURB-65 score of 3 and 25\% of patients with CURB-65 score of 4 . Mortality was observed to be higher in patients with CURB-65 score of 2 since the patients with this score had more comorbid conditions compared to those with higher CURB-65 scores. Such findings highlight the disadvantage of using CURB65 score as a severity assessment tool since it does not account for comorbid conditions of patients which one of the main factors associated with the outcomes in CAP. With the CURB-65 score, the age cut off limit of $\geq 65$ years results in underestimation of the severity of the illness in those who fall behind this age limit even though they have substantial mortality risks and overestimation of severity of illness in the elderly patients who may not have substantial risk factors for mortality other than their age.

When we assess the predictive ability of the CURB-65 score to predict mortality as an outcome, CURB-65 score had a sensitivity of $87.5 \%$, specificity of $43.6 \%$, PPV of $11.7 \%$ and NPV of $97.6 \%$. These findings were similar to findings reported from studies in India and Iran ${ }^{11,19}$. In this study the interval of confidence is wider because of the small sample size which is one of the limitations of the study.

Other limitations of the study is, this was a single center non randomized study with a small sample size. The true burden of CAP may have been underestimated due to exclusion of patients with missing data.

Risk factors other than the age and comorbidities of the patients which are associated with mortality were not evaluated in this study.

When considering the use of CURB-65 score as a severity assessment tool, we must take into consideration the factors such as comorbid conditions and age of the patient which are associated with the outcomes of CAP. With the advantage of its simplicity, practicality and time-efficiency, the CURB-65 score can be used as a supplement to clinical judgement as a severity assessment tool in our hospital settings.

\section{CONCLUSIONS}

CAP is more prevalent in the middle age and elderly group, and majority of the patients had at least one comorbid condition with the most common comorbidities being cardiovascular and lung diseases in this study. These findings highlight the importance of appropriate risk reduction interventions and prompt management of the disease to reduce the complications and burden of CAP.

With the use of CURB-65 score as a severity assessment tool, $41.2 \%$ of patients in this study who were in the low-risk group received inpatient care, had these patients been considered for home-based care management would have resulted in reduced burden and exhaustion of resources in our hospital setting.

In this study CURB-65 score had a high sensitivity and negative predictive value in predicting death as an outcome of patients with CAP which validates the usefulness of CURB-65 score as a severity assessment tool and can be integrated into clinical practice in our hospital setting. However, factors such as comorbidities and development of complications of pneumonia which are associated with risk of mortality in patients with CAP should be considered.

\section{RECOMMENDATIONS}

From the findings of this study, preventive interventions and appropriate treatment must be targeted to the middle age and the elderly populations with comorbidities which would reduce the burden and implications of CAP on the health care system.

CURB-65 score can be used as a supplement to clinical judgment in assessing the severity of CAP and to predict mortality as an outcome in clinical practice.

With the help of CURB-65 score, patients can be classified under low-risk group and considered for homebased care management which would minimize unnecessary hospital admissions and reduce the burden on the resources in the health care system. Using the CURB-65 score, the site of care can be considered and recommendations from the national antibiotic guidelines for empirical treatment can be implemented accordingly.

\section{REFERENCES}

1. Abbafati C, Abbas KM, Abbasi KM, Abd-Allah F, Abdelalim A, Abdollahi M, et al. Global burden of 369 diseases and injuries in 204 countries and territories, 1990-2019: a systematic analysis for the Global Burden of Disease Study

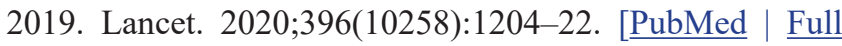
$\underline{\text { Text }} \mid \underline{\text { DOI] }}$ 
2. Azmi S, Aljunid S, Maimaiti N, Ali A, Muhammad N, De R, et al. Assessing the burden of pneumonia using administrative data from Malaysia, Indonesia, and the Philippines. Int J Infect Dis. 2016;49:87-93.[PubMed $\mid$ Full Text $\mid$ DOI]

3. Song JH, Oh WS, Kang CI, Chung DR, Peck KR, Ko KS, et al. Epidemiology and clinical outcomes of community-acquired pneumonia in adult patients in Asian countries: a prospective study by the Asian network for surveillance of resistant pathogens. Int J Antimicrob Agents. 2008;31(2):107-14. [PubMed | Full Text | DOI]

4. Ministry of Health. Annual health bulletin . Ministry of Health,Bhutan; 2020; 1-122 p.

5. Lim WS, Van Der Eerden MM, Laing R, Boersma WG, Karalus N, Town GI, et al. Defining community acquired pneumonia severity on presentation to hospital: An international derivation and validation study. Thorax. 2003;58(5):377-82. [PubMed | Full Text | DOI]

6. National Institute for Health and Care Excellence (NICE). Pneumonia in adults: diagnosis and management. Nice. 2018;(December 2014):1-18. [Full Text]

7. Carter JV, Pan J, Rai SN, Galandiuk S. ROC-ing along: Evaluation and interpretation of receiver operating characteristic curves. Surg (United States). 2016;159(6):1638-45. [PubMed | Full Text | DOI]

8. Mandrekar JN. Simple statistical measures for diagnostic accuracy assessment. J Thorac Oncol. 2010;5(6):763-4. [PubMed | Full Text | DOI]

9. Rivero-Calle I, Pardo-Seco J, Aldaz P, Vargas DA, Mascarós $\mathrm{E}$, Redondo $\mathrm{E}$, et al. Incidence and risk factor prevalence of community-acquired pneumonia in adults in primary care in Spain (NEUMO-ES-RISK project). [PubMed | Full Text | DOI]

10. Torres A, Peetermans WE, Viegi G, Blasi F. Risk factors for community-acquired pneumonia in adults in Europe: A literature review. Thorax. 2013;68(11):1057-65. [PubMed | Full Text $\mid$ DOI]

11. Babu A, Jose N, Jose J. A prospective observational study to evaluate the severity assessment scores in communityacquired pneumonia for adult patients. Indian J Respir Care. 2017;6(2):820. [Full Text | DOI]
12. Gebru HB, Gahse FE, Kahsay AB. Risk factors of community acquired pneumonia among adults in Tigray, Ethiopia: A case-control study. J Clin Diagnostic Res. 2018;12(5):LG015. [Full Text $\mid$ DOI]

13. Almirall J, Serra-Prat M, Bolíbar I, Balasso V. Risk Factors for Community-Acquired Pneumonia in Adults: A Systematic Review of Observational Studies. Respiration. 2017;94(3):299-311. [PubMed | Full Text | DOI]

14. Metlay J, Waterer G, Long A, Anzueto A, Brozek J, Crothers $\mathrm{K}$, et al. AMERICAN THORACIC SOCIETY Diagnosis and Treatment of Adults with Community-acquired Pneumonia An Of fi cial Clinical Practice Guideline of the American Thoracic Society and Infectious Diseases Society of America. 2019;200. [PubMed | Full Text | DOI]

15. Bjarnason A, Westin J, Lindh M, Andersson LM, Kristinsson $\mathrm{KG}$, Löve A, et al. Incidence, etiology, and outcomes of community-acquired pneumonia: A population-based study. Open Forum Infect Dis. 2018 Feb;5(2):ofy010. [PubMed | Full Text | DOI]

16. Zhang ZX, Yong Y, Tan WC, Shen L, Ng HS, Fong KY. Prognostic factors for mortality due to pneumonia among adults from different age groups in Singapore and mortality predictions based on PSI and CURB-65. Singapore Med J. 2018;59(4):190-8. [PubMed | Full Text | DOI]

17. Suter-Widmer I, Christ-Crain M, Zimmerli W, Albrich W, Mueller B, Schuetz P, et al. Predictors for length of hospital stay in patients with community-acquired Pneumonia: Results from a Swiss Multicenter study. BMC Pulm Med. 2012;12(1):1.[Full Text | DOI]

18. Loke YK, Kwok CS, Niruban A, Myint PK. Value of severity scales in predicting mortality from community-acquired pneumonia: Systematic review and meta-analysis. Thorax. 2010;65(10):884-90. [PubMed | Full Text | DOI]

19. Alavi-Moghaddam M, Bakhshi H, Rezaei B, Khashayar P. Pneumonia severity index compared to CURB-65 in predicting the outcome of community acquired pneumonia among patients referred to an Iranian emergency department: A prospective survey. Brazilian J Infect Dis. 2013;17(2):179_ 83. [PubMed $\mid$ Full Text $\mid$ DOI]

20. Ministry of Health.National Antibiotic Guidelines. Ministry of Health,Bhutan;2018

\section{AUTHORS CONTRIBUTION}

Following authors have made substantial contributions to the manuscript as under:

SC: Concept, design, data collection and analysis, manuscript writing

CLA: Concept, design, manuscript writing and review

DS: Concept, manuscript writing and review

SCR: Concept, manuscript writing and review

Author agree to be accountable for all respects of the work in ensuring that questions related to the accuracy and integrity of any part of the work are appropriately investigated and resolved.

\section{CONFLICT OF INTEREST}

None

GRANT SUPPORT AND FINANCIAL DISCLOSURE

Ministry of Health, Royal Government of Bhutan 